\title{
THE $q$-SELBERG POLYNOMIALS FOR $n=2$
}

\author{
KEVIN W. J. KADELL
}

\begin{abstract}
We have conjectured that Selberg's integral has a plethora of extensions involving the Selberg polynomials and proved that these are the Schur functions for $k=1$. We prove this conjecture for $n=2$ and show that the polynomials are, in a formal sense, Jacobi polynomials. We conjecture an orthogonality relation for the Selberg polynomials which combines orthogonality relations for the Schur functions and Jacobi polynomials. We extend a basic Schur function identity.

We give a $q$-analogue of the Selberg polynomials for $n=2$ using the little $q$-Jacobi polynomials.
\end{abstract}

1. Introduction and summary. Fix $n \geq 2, k \geq 0$. We conjecture [12] that for each partition $\lambda=\left(\lambda_{1}, \lambda_{2}, \ldots, \lambda_{n}\right), \lambda_{1} \geq \lambda_{2} \geq \cdots \geq \lambda_{n} \geq 0$, there is a homogeneous symmetric polynomial $s_{n, \lambda}^{k}(t)=s_{n, \lambda}^{k}\left(t_{1}, \ldots, t_{n}\right)$ with leading term $\prod_{i=1}^{n} t_{i}^{\lambda_{i}}$ such that

$$
\begin{gathered}
\int_{0}^{1} \cdots \int_{0}^{1} \prod_{i=1}^{n} t_{i}^{(x-1)}\left(1-t_{i}\right)^{(y-1)} s_{n, \lambda}^{k}(\mathbf{t}) \prod_{1 \leq i<j \leq n}\left(t_{i}-t_{j}\right)^{2 k} d t_{1} \cdots d t_{n} \\
=n ! f_{n, \lambda}^{k} \prod_{i=1}^{n} \frac{\Gamma\left(x+(n-i) k+\lambda_{i}\right) \Gamma(y+(n-i) k)}{\Gamma\left(x+y+(2 n-i-1) k+\lambda_{i}\right)}
\end{gathered}
$$

where, as holds throughout, $\operatorname{Re}(x)>0, \operatorname{Re}(y)>0$,

$$
f_{n, \lambda}^{k}=\prod_{1 \leq i<j \leq n}\left(\lambda_{i}-\lambda_{j}+k(j-i)\right)_{k},
$$

and

$$
(a)_{0}=1, \quad(a)_{m}=\prod_{i=0}^{m-1}(a+i), \quad m \geq 1 .
$$

We call $s_{n, \lambda}^{k}(\mathbf{t})$ the Selberg polynomials since the case $\lambda=(0, \ldots, 0)$ is Selberg's integral $[\mathbf{1 5}]$. We need only determine them for $\lambda_{n}=0$ since we may set

$$
s_{n,\left(\lambda_{1}, \ldots, \lambda_{n}\right)}^{k}(\mathbf{t})=\left(t_{1} \cdots t_{n}\right)^{\lambda_{n}} s_{n,\left(\lambda_{1}-\lambda_{n}, \ldots, \lambda_{n-1}-\lambda_{n}, 0\right)}^{k}(\mathbf{t}) .
$$

Received by the editors June 30, 1986.

1980 Mathematics Subject Classification (1985 Revision). Primary 33A15.

The research for this paper was done while on a visiting appointment during 1985-1986 to the Department of Combinatorics and Optimization, University of Waterloo, Ontario, Canada N2L $3 \mathrm{G} 1$.

Research supported by NSERC Grants A8907 and A8235. 
It is clear that for $k=0$ the Selberg polynomials are the monomial symmetric functions. To compute $f_{n, \lambda}^{0}$, express $f_{n, \lambda}^{k}(1.2)$ in terms of gamma functions and take the limit as $k$ tends to 0 . In [12], we showed that for $k=1$

$$
s_{n, \boldsymbol{\lambda}}^{1}(\mathbf{t})=\frac{\operatorname{det}\left|t_{j}^{n-i+\lambda_{i}}\right|_{n \times n}}{\operatorname{det}\left|t_{j}^{n-i}\right|_{n \times n}}
$$

the Selberg polynomials are Schur functions. We conjectured that there are $q$ Selberg polynomials which satisfy a $q$-analogue of (1.1) and obtained some $q$ analogues of (1.5). Aomoto [2] has shown that

$$
s_{n,\left(1^{m}\right)}^{k}(\mathbf{t})=\sum_{1 \leq i_{1}<i_{2}<\cdots<i_{m} \leq n} t_{i_{1}} \cdots t_{i_{m}},
$$

where $\left(1^{m}\right)$ denotes the partition $\lambda_{1}=\cdots=\lambda_{m}=1, \lambda_{m+1}=\cdots=\lambda_{n}=0$. Thus the Selberg polynomials include the elementary symmetric functions for all $k \geq 0$. Kadell [13] extends Aomoto's argument to give a $q$-analogue of (1.6). Habsieger [9] treats the case $m=0$ by extending Selberg's proof.

Our main result is

THEOREM 1. For $k \geq 1$,

$$
s_{2,(\lambda, 0)}^{k}(s, t)=\sum_{i=0}^{\lambda} \frac{(-\lambda)_{i}(k)_{i}}{i !(1-\lambda-k)_{i}} s^{i} t^{\lambda-i} .
$$

Aomoto [2] uses (1.6) to give an integral representation of the Jacobi polynomials (see Szegö $[\mathbf{1 7},(4.21 .2)]$ )

$$
p_{n}^{(x, y)}(t)=\sum_{i=0}^{n} \frac{(-n)_{i}(n+x+y-1)_{i}}{i !(x)_{i}} t^{i}
$$

These are orthogonal on $(0,1)$ with respect to the beta distribution

$$
\int_{0}^{1} t^{(x-1)}(1-t)^{(y-1)} d t=\frac{\Gamma(x) \Gamma(y)}{\Gamma(x+y)}
$$

which is the case $n=1$ of Selberg's integral. (1.7) then becomes

$$
s_{2,(\lambda, 0)}^{k}(s, t)=t^{\lambda} p_{\lambda}^{(1-\lambda-k, 2 k)}(s / t), \quad k \geq 1 .
$$

We have

$$
\begin{aligned}
s_{2,(\lambda, 0)}^{0}(s, t) & =t^{\lambda}+s^{\lambda} \chi(\lambda>0) \\
& =t^{\lambda} \lim _{\varepsilon \rightarrow 0} p_{\lambda}^{(1-\lambda+\varepsilon, 2 \varepsilon)}(s / t),
\end{aligned}
$$

where $\chi(A)$ is 1 or 0 according to whether $A$ is true or false. Thus, for $n=2$ the Selberg polynomials are, in a formal sense, Jacobi polynomials.

In $\S 2$, we obtain a difference equation for the Selberg polynomials when $n=$ 2. We express the orthogonality of the Jacobi polynomials as a rational function identity. In $\S 3$, we use this to prove Theorem 1.

In $\S 4$, we interpret this orthogonality as a constant term identity. We conjecture an orthogonality relation which combines orthogonality relations for the Schur functions and Jacobi polynomials. 
In $\S 5$, we extend the well-known Schur function identity (see Stanley [16])

$$
\sum_{\lambda} s_{n, \lambda}^{1}(\mathbf{t})=\prod_{i=1}^{n} \frac{1}{\left(1-t_{i}\right)} \prod_{1 \leq i<j \leq n} \frac{1}{\left(1-t_{i} t_{j}\right)} .
$$

In $\S 6$, we recall a $q$-analogue of the beta integral (1.9) and the orthogonal polynomials for this measure. These are called the little $q$-Jacobi polynomials. We obtain a $q$-difference equation for the $q$-Selberg polynomials when $n=2$.

In $\S 7$, we use the orthogonality of the little $q$-Jacobi polynomials to prove a $q$ analogue of Theorem 1 (1.7). This shows that for $n=2$ the $q$-Selberg polynomials are, in a formal sense, little $q$-Jacobi polynomials.

There are many measures $d F(t)$ which provide $q$-analogues of the beta integral (1.9) and for which there is a conjectured $q$-analogue of Selberg's integral. See Askey [4] and Rahman [14]. In [12], we were able to treat the case $k=1$ by a method which seems to work for many of these measures. It is related to the polynomials $\left\{P_{n}(t) \mid n \geq 0\right\}$ which are orthogonal with respect to $d F(t)$. In $\S 8$, we suggest that Theorem 1 (1.7) can be extended using the orthogonality of $\left\{P_{n}(t) \mid n \geq 0\right\}$.

2. A difference equation. The basic technique for finding relations among the Selberg polynomials is to multiply a potential relation by

$$
W_{n}(x, y, k)=\prod_{i=1}^{n} t_{i}^{(x-1)}\left(1-t_{i}\right)^{(y-1)} \prod_{1 \leq i<j \leq n}\left(t_{i}-t_{j}\right)^{2 k}
$$

and integrate. This gives a relation among gamma functions for which, hopefully, we may determine the coefficients. Set $s=t_{1}, t=t_{2}$. By (1.1), we have

$$
\begin{aligned}
& \int_{0}^{1} \int_{0}^{1}(s-t)^{2} s_{2,(\lambda, 0)}^{k+1}(s, t) W_{2}(x, y, k) d s d t \\
& \quad=2(\lambda+k+1)_{(k+1)} \frac{\Gamma(x) \Gamma(x+k+\lambda+1) \Gamma(y) \Gamma(y+k+1)}{\Gamma(x+y+k+1) \Gamma(x+y+2 k+\lambda+2)} .
\end{aligned}
$$

We want to express $(s-t)^{2} s_{2,(\lambda, 0)}^{k+1}(s, t)$ as a linear combination of Selberg polynomials corresponding to the exponent $k$. The leading term $t^{\lambda+2}$ is the same as that of $s_{2,(\lambda+2,0)}^{k}(s, t)$. By $(1.1)$, we have

$$
\begin{aligned}
& \int_{0}^{1} \int_{0}^{1} s_{2,(\lambda+2,0)}^{k}(s, t) W_{2}(x, y, k) d s d t \\
& \quad=2(\lambda+k+2)_{k} \frac{\Gamma(x) \Gamma(x+k+\lambda+2) \Gamma(y) \Gamma(y+k)}{\Gamma(x+y+k) \Gamma(x+y+2 k+\lambda+2)} .
\end{aligned}
$$

Hence

$$
\begin{aligned}
\int_{0}^{1} \int_{0}^{1}\left[s_{2,(\lambda+2,0)}^{k}(s, t)-(s-t)^{2} s_{2,(\lambda, 0)}^{k+1}(s, t)\right] W_{2}(x, y, k) d s d t \\
=2(\lambda+k+2)_{k} \frac{\Gamma(x) \Gamma(x+k+\lambda+1) \Gamma(y) \Gamma(y+k)}{\Gamma(x+y+k+1) \Gamma(x+y+2 k+\lambda+2)} \\
\cdot[(x+k+\lambda+1)(x+y+k)-(\lambda+k+1)(y+k)] \\
=2(\lambda+k+2)_{k} \frac{\Gamma(x+1) \Gamma(x+k+\lambda+1) \Gamma(y) \Gamma(y+k)}{\Gamma(x+y+k+1) \Gamma(x+y+2 k+\lambda+1)} .
\end{aligned}
$$


But, (1.1) gives

$$
\begin{aligned}
& \int_{0}^{1} \int_{0}^{1} s t s_{2,(\lambda, 0)}^{k}(s, t) W_{2}(x, y, k) d s d t \\
& \quad=2(\lambda+k)_{k} \frac{\Gamma(x+1) \Gamma(x+k+\lambda+1) \Gamma(y) \Gamma(y+k)}{\Gamma(x+y+k+1) \Gamma(x+y+2 k+\lambda+1)} .
\end{aligned}
$$

Comparing (2.4) and (2.5), we have

$$
\begin{gathered}
\int_{0}^{1} \int_{0}^{1}\left[s_{2,(\lambda+2,0)}^{k}(s, t)-(s-t)^{2} s_{2,(\lambda, 0)}^{k+1}(s, t)\right] W_{2}(x, y, k) d s d t \\
=\frac{(\lambda+2 k)_{2}}{(\lambda+k)_{2}} \int_{0}^{1} \int_{0}^{1} s t s_{2,(\lambda, 0)}^{k}(s, t) W_{2}(x, y, k) d s d t .
\end{gathered}
$$

Equating integrands and rearranging gives

$$
\begin{aligned}
& (\lambda+2 k)_{2} s t s_{2,(\lambda, 0)}^{k}(s, t) \\
& \quad=(\lambda+k)_{2}\left[s_{2,(\lambda+2,0)}^{k}(s, t)-(s-t)^{2} s_{2,(\lambda, 0)}^{k+1}(s, t)\right] .
\end{aligned}
$$

We wish to interpret $s_{2,(\lambda, 0)}^{k}(s, t)$ as a Jacobi polynomial via $(1.10)$ and relate (2.7) to the orthogonality. Divide both sides of $(2.7)$ by $(\lambda+2 k)_{2} s t^{\lambda+1}$ and replace $s / t$ by $t$. Using (1.10), (2.7) becomes

$$
\begin{aligned}
p_{\lambda}^{(1-\lambda-k, 2 k)}(t)= & \frac{(\lambda+k)_{2}}{(\lambda+2 k)_{2}} \frac{1}{t} p_{\lambda+2}^{(-1-\lambda-k, 2 k)}(t) \\
& -\frac{(\lambda+k)_{2}}{(\lambda+2 k)_{2}} \frac{1}{t}(1-t)^{2} p_{\lambda}^{(-\lambda-k, 2 k+2)}(t)
\end{aligned}
$$

The orthogonality of the Jacobi polynomials (1.8) is given by

$$
\int_{0}^{1} t^{m} p_{n}^{(x, y)}(t) t^{(x-1)}(1-t)^{(y-1)} d t=0, \quad 0 \leq m<n .
$$

Set

$$
p_{n}^{(x, y)}(t)=\sum_{i=0}^{n} c_{n}^{(x, y)}(i) t^{i}
$$

The coefficients

$$
c_{n}^{(x, y)}(i)=\frac{(-n)_{i}(n+x+y-1)_{i}}{i !(x)_{i}}
$$

are uniquely determined by the orthogonality (2.9) and the normalization

$$
c_{n}^{(x, y)}(0)=1 \text {. }
$$

Using the well-known functional equation

$$
\Gamma(x+1)=x \Gamma(x),
$$


we have

$$
\begin{aligned}
\int_{0}^{1} t^{m} & p_{n}^{(x, y)}(t) t^{(x-1)}(1-t)^{(y-1)} d t \\
& =\sum_{i=0}^{n} c_{n}^{(x, y)}(i) \int_{0}^{1} t^{(x+m+i-1)}(1-t)^{(y-1)} d t \\
& =\sum_{i=0}^{n} c_{n}^{(x, y)}(i) \frac{\Gamma(x+m+i) \Gamma(y)}{\Gamma(x+y+m+i)} \\
& =\frac{\Gamma(x+m) \Gamma(y)}{\Gamma(x+y+m+n)} \sum_{i=0}^{n} c_{n}^{(x, y)}(i)(x+m)_{i}(x+y+m+i)_{n-i}
\end{aligned}
$$

Thus, the orthogonality (2.9) becomes

$$
\sum_{i=0}^{n} c_{n}^{(x, y)}(i)(x+m)_{i}(x+y+m+i)_{n-i}=0, \quad 0 \leq m<n .
$$

Substituting (2.11) into (2.15), we see that both sides of (2.15) are rational functions of $x$ and $y$. Thus, (2.15) holds for all complex $x$ and $y$.

3. A proof of Theorem 1. We have shown that if two of the three Selberg polynomials appearing in (2.7) satisfy (1.1), then so does the third. To prove Theorem 1 , we may show that (1.7) satisfies (2.7) and, since only one of the polynomials in (2.7) involves the exponent $k+1$, we may use induction $k$. We use (2.8) rather than (2.7).

Observe that (1.5) gives

$$
s_{2,(\lambda, 0)}^{1}(s, t)=\frac{\operatorname{det}\left|\begin{array}{cc}
s^{\lambda+1} & t^{\lambda+1} \\
1 & 1
\end{array}\right|}{\operatorname{det}\left|\begin{array}{ll}
s & t \\
1 & 1
\end{array}\right|}=\frac{\left(s^{\lambda+1}-t^{\lambda+1}\right)}{(s-t)}=\sum_{i=0}^{\lambda} s^{i} t^{\lambda-i} .
$$

Thus (1.7) holds for $k=1$.

We now show that for $k \geq 1$, the right side of (2.8) is a polynomial of degree at most $\lambda$ which satisfies the orthogonality (2.15) with

$$
x=1-\lambda-k, \quad y=2 k, \quad n=\lambda,
$$

and the normalization (2.12). Let $a$ be an integer with $\operatorname{Re}(x+a)>0$ and $b$ be a nonnegative integer. Then

$$
\int_{0}^{1} t^{m}\left(t^{a}(1-t)^{b} p_{n}^{(x+a, y+b)}(t)\right) t^{(x-1)}(1-t)^{(y-1)} d t=0, \quad 0 \leq m<n .
$$

Set

$$
t^{a}(1-t)^{b} p_{n}^{(x+a, y+b)}(t)=\sum_{i=a}^{a+b+n} d_{n,(a, b)}^{(x, y)}(i) t^{i}
$$

The orthogonality (3.3) becomes

$$
\sum_{i=a}^{a+b+n} d_{n,(a, b)}^{(x, y)}(i)(x+m+a)_{(i-a)}(x+y+m+i)_{(n+a+b-i)}=0, \quad 0 \leq m<n
$$


Setting

$$
x=1-\lambda-k, \quad y=2 k, \quad n=\lambda, \quad a=-1, \quad b=2,
$$

in (3.5), we have

$$
\begin{aligned}
\sum_{i=-1}^{\lambda+1} d_{\lambda,(-1,2)}^{(1-\lambda-k, 2 k)}(i)(-\lambda-k+m)_{(i+1)}(1-\lambda+k+m+i)_{(\lambda+1-i)} & =0 \\
0 & \leq m<\lambda
\end{aligned}
$$

Using

$$
x=-\lambda-k, \quad y=2 k, \quad n=\lambda+2, \quad a=-1, \quad b=0,
$$

we obtain

$$
\begin{array}{r}
\sum_{i=-1}^{\lambda+1} d_{\lambda+2,(-1,0)}^{(-\lambda-k, 2 k)}(i)(-1-\lambda-k+m)_{(i+1)}(-\lambda+k+m+i)_{(\lambda+1-i)}=0 \\
0 \leq m<\lambda+2 .
\end{array}
$$

If we replace $m$ by $m+1$ in (3.9), we obtain an orthogonality with the same coefficients as in (3.7) which is valid for $-1 \leq m<\lambda+1$. Let

$$
\text { right side of }(2.8)=\sum_{i=-1}^{\lambda+1} e(i) t^{i} \text {. }
$$

Then we have

$$
\sum_{i=-1}^{\lambda+1} e(i)(-\lambda-k+m)_{(i+1)}(1-\lambda+k+m+i)_{(\lambda+1-i)}=0, \quad 0 \leq m<\lambda .
$$

Using

$$
(\alpha)_{i}=(-1)^{i}(1-i-\alpha)_{i}
$$

we have, for $k \geq 1$,

$$
\begin{aligned}
\frac{(-\lambda)_{\lambda-i}(k)_{\lambda-i}}{(\lambda-i) !(1-\lambda-k)_{\lambda-i}} & =\frac{(-\lambda)_{\lambda}(k)_{\lambda}}{\lambda !(1-\lambda-k)_{\lambda}} \frac{(1+\lambda-i)_{i}(1-k-i)_{i}}{(-i)_{i}(k+\lambda-i)_{i}} \\
& =\frac{(-\lambda)_{i}(k)_{i}}{i !(1-k-\lambda)_{i}} .
\end{aligned}
$$

Thus the polynomials defined by (1.7) are symmetric in $s$ and $t$. We have

$$
\begin{gathered}
e(i)=e(\lambda-i), \quad-1 \leq i \leq \lambda+1, \\
e(-1)=e(\lambda+1)=0 .
\end{gathered}
$$

By (3.14), the sum in (3.11) may be taken with $i$ running from 0 to $\lambda$. Dividing by $(-\lambda-k+m)(1+k+m)$, we obtain

$$
\sum_{i=0}^{\lambda} e(i)(1-\lambda-k+m)_{i}(1-\lambda+k+m+i)_{(\lambda-i)}=0, \quad 0 \leq m<\lambda .
$$


Thus the right side of (2.8) is a polynomial of degree at most $\lambda$ which satisfies the orthogonality relation (2.15) with the parameters given by (3.2). By (2.15), the left side of (2.8) also satisfies (3.15). A simple computation

$$
e(0)=\frac{(\lambda+k)_{2}}{(\lambda+2 k)_{2}}\left[\frac{(\lambda+2) k}{(1+\lambda+k)}-\frac{\lambda(k+1)}{(\lambda+k)}+2\right]=1
$$

gives the normalization (2.12).

To complete the proof of Theorem 1, we must show that the system of equations (3.15) and the normalization (3.16) uniquely determine $p_{\lambda}^{(1-\lambda-k, 2 k)}(t)$. Since the parameters (3.2) do not correspond to an integrable measure, we cannot rely on the usual argument. We evaluate the determinant of the coefficient matrix. Substituting (2.12) into (2.15) gives a system of equations with coefficient matrix

$$
C_{n}^{(x, y)}=\left|(x+i)_{j}(x+y+i+j)_{n-j}\right|_{0 \leq i<n, 1 \leq j \leq n} .
$$

For $1 \leq s \leq n$, set

$$
C_{n, s}^{(x, y)}=\left|c_{n, s}^{(x, y)}(i, j)\right|_{0 \leq i<n, 1 \leq j \leq n}
$$

where

$$
c_{n, s}^{(x, y)}(i, j)= \begin{cases}(x+i)_{j}(x+y+i+j+n-s)_{s-j}, & 0 \leq i<n, 1 \leq j \leq s \\ (x+i)_{j}, & 0 \leq i<n, s<j \leq n\end{cases}
$$

Then we have

$$
C_{n}^{(x, y)}=C_{n, n}^{(x, y)}
$$

Observe that

$$
\begin{aligned}
& (x+i)_{j}(x+y+i+j+n-s)_{s-j} \\
& \quad-(x+i)_{j+1}(x+y+i+j+n-s+1)_{s-j-1} \\
& =(y+n-s)(x+i)_{j}(x+y+i+j+n-s+1)_{s-j-1} .
\end{aligned}
$$

Using (3.21), we may subtract column $j+1$ from column $j$ and divide by $(y+n-s)$ where $j$ runs from 1 to $s-1$. This gives

$$
\operatorname{det}\left(C_{n, s}^{(x, y)}\right)=(y+n-s)^{(s-1)} \operatorname{det}\left(C_{n, s-1}^{(x, y)}\right), \quad 2 \leq s \leq n .
$$

Observe that

$$
C_{n, 1}^{(x, y)}=\left|(x+i)_{j}\right|_{0 \leq i<n, 1 \leq j \leq n}
$$

does not depend on $y$. To obtain the dependence on $x$, divide row $i$ by $x+i$ for $0 \leq i<n$. This gives

$$
\operatorname{det}\left(C_{n, 1}^{(x, y)}\right)=(x)_{n} \operatorname{det}\left(D_{n}^{(x, y)}\right)
$$

where

$$
D_{n}^{(x, y)}=\left|(x+i+1)_{j-1}\right|_{0 \leq i<n, 1 \leq j \leq n} .
$$


To evaluate $\operatorname{det}\left(D_{n}^{(x, y)}\right)$, subtract row $i-1$ from row $i$ where $i$ runs from $n-1$ to 1 and expand along column 1 . We obtain

$$
\begin{aligned}
\operatorname{det}\left(D_{n}^{(x, y)}\right) & =\operatorname{det}\left|\begin{array}{ccccc}
1 & x+1 & (x+1)_{2} & \ldots & (x+1)_{n-1} \\
1 & x+2 & (x+2)_{2} & \ldots & (x+2)_{n-1} \\
\vdots & \vdots & \vdots & & \vdots \\
1 & x+n & (x+n)_{2} & \ldots & (x+n)_{n-1}
\end{array}\right|_{n \times n} \\
& =\operatorname{det}\left|\begin{array}{ccccc}
1 & x+1 & (x+1)_{2} & \ldots & (x+1)_{n-1} \\
0 & 1 & 2(x+2) & \ldots & (n-1)(x+2)_{n-2} \\
\vdots & \vdots & \vdots & & \vdots \\
0 & 1 & 2(x+n) & \ldots & (n-1)(x+n)_{n-2}
\end{array}\right|_{n \times n} \\
& =(n-1) ! \operatorname{det}\left(D_{n-1}^{(x+1, y)}\right) .
\end{aligned}
$$

Combining our results, we obtain

$$
\operatorname{det}\left(C_{n}^{(x, y)}\right)=(x)_{n} \prod_{s=2}^{n}(s-1) !(y+n-s)^{(s-1)} .
$$

Substituting (3.2) into (3.27) gives

$$
\operatorname{det}\left(C_{\lambda}^{(1-\lambda-k, 2 k)}\right)=(1-\lambda-k)_{\lambda} \prod_{s=2}^{\lambda}(s-1) !(2 k+\lambda-s)^{(s-1)} .
$$

The proof of Theorem 1 is now completed by observing that for $k \geq 1$ this is not 0 .

4. Orthogonality. The left side of (2.15) is a polynomial in $m$ of degree at most $n$ which vanishes for $0 \leq m<n$. Hence

$$
\sum_{i=0}^{n} \frac{(-n)_{i}(n+x+y-1)_{i}}{i !(x)_{i}}(x+m)_{i}(x+y+m+i)_{n-i}=c(m+1-n)_{n} .
$$

To evaluate $c$, we require the Chu-Vandermonde sum (see Bailey $[\mathbf{7}, \S 1.3]$ ). It is

$$
\sum_{i=0}^{n} \frac{(-n)_{i}(b)_{i}}{i !(c)_{i}}=\frac{(c-b)_{n}}{(c)_{n}} .
$$

Equating coefficients of $m^{n}$ in (4.1) gives

$$
c=\sum_{i=0}^{n} \frac{(-n)_{i}(n+x+y-1)_{i}}{i !(x)_{i}}=\frac{(1-n-y)_{n}}{(x)_{n}} .
$$

Thus (4.1) becomes

$$
\begin{aligned}
& \sum_{i=0}^{n} \frac{(-n)_{i}(n+x+y-1)_{i}}{i !(x)_{i}}(x+m)_{i}(x+y+m+i)_{n-i} \\
& \quad=\frac{(1-n-y)_{n}}{(x)_{n}}(m+1-n)_{n} .
\end{aligned}
$$

This is equivalent to Saalschütz's sum (see Bailey [7, §2.2]). This is

$$
\sum_{i=0}^{n} \frac{(-n)_{i}(a)_{i}(b)_{i}}{i !(c)_{i}(1+a+b-c-n)_{i}}=\frac{(c-a)_{n}(c-b)_{n}}{(c)_{n}(c-a-b)_{n}} .
$$


It is well-known that (4.5) gives the orthogonality (2.9) of the Jacobi polynomials.

For the parameters given in (3.2), we have

$$
c=\frac{(1-\lambda-2 k)_{\lambda}}{(1-\lambda-k)_{\lambda}}=\frac{(2 k)_{\lambda}}{(k)_{\lambda}}=\frac{(\lambda+k)_{k}}{(k)_{k}} .
$$

Thus (4.4) gives

$$
\begin{aligned}
& \sum_{i=0}^{\lambda} \frac{(-\lambda)_{i}(k)_{i}}{i !(1-\lambda-k)_{i}}(1-\lambda-k+m)_{i}(1-\lambda+k+m+i)_{\lambda-i} \\
& \quad=\frac{(\lambda+k)_{k}}{(k)_{k}}(m+1-\lambda)_{\lambda} .
\end{aligned}
$$

Let $[w] f$ denote the coefficient of $w$ in the Laurent expansion of $f$ where $w$ is a monomial. Observe that

$$
\begin{aligned}
\left(1-\frac{t}{s}\right)^{k-1}\left(1-\frac{s}{t}\right)^{k} & =\left(-\frac{t}{s}\right)^{k-1}\left(1-\frac{s}{t}\right)^{2 k-1} \\
& =\left(-\frac{t}{s}\right)^{k-1} \sum_{i=0}^{2 k-1} \frac{(2 k-1) !}{i !(2 k-1-i) !}\left(-\frac{s}{t}\right)^{i} \\
& =\sum_{i=0}^{2 k-1} \frac{(2 k-1) !}{i !(2 k-1-i) !}\left(-\frac{s}{t}\right)^{(i+1-k)} .
\end{aligned}
$$

This gives

$$
\text { [1] }\left(\frac{s}{t}\right)^{a}\left(1-\frac{t}{s}\right)^{k-1}\left(1-\frac{s}{t}\right)^{k}=(-1)^{a} \frac{(2 k-1) !}{(k-1-a) !(k+a) !} .
$$

Using (4.7) and (4.9), we obtain

$$
\begin{aligned}
{[1] s^{m-\lambda} t^{-m} s_{2,(\lambda, 0)}^{k}(s, t)\left(1-\frac{t}{s}\right)\left(1-\frac{s}{t}\right)^{k} } \\
=\sum_{i=0}^{\lambda} \frac{(-\lambda)_{i}(k)_{i}}{i !(1-\lambda-k)_{i}}[1]\left(\frac{s}{t}\right)^{m-\lambda+i}\left(1-\frac{t}{s}\right)^{k-1}\left(1-\frac{s}{t}\right)^{k} \\
=\sum_{i=0}^{\lambda} \frac{(-\lambda)_{i}(k)_{i}}{i !(1-\lambda-k)_{i}}(-1)^{m-\lambda+i} \frac{(2 k-1) !}{(k-1+\lambda-m-i) !(k-\lambda+m+i) !} \\
=\frac{(-1)^{m-\lambda}(2 k-1) !}{(k+m) !(k-1+\lambda-m) !} \\
\quad \cdot \sum_{i=0}^{\lambda} \frac{(-\lambda)_{i}(k)_{i}}{i !(1-\lambda-k)_{i}}(1-\lambda-k+m)_{i}(1-\lambda+k+m+i)_{\lambda-i} \\
=\frac{(-1)^{m-\lambda}(2 k-1) !}{(k+m) !(k-1+\lambda-m) !} \frac{(\lambda+k)_{k}}{(k)_{k}}(m+1-\lambda)_{\lambda} .
\end{aligned}
$$

This gives the orthogonality

$$
[1] s^{m-\lambda} t^{-m} s_{2,(\lambda, 0)}^{k}(s, t)\left(1-\frac{t}{s}\right)^{k-1}\left(1-\frac{s}{t}\right)^{k}=0, \quad 0 \leq m<\lambda .
$$


The constant term is also trivially 0 when $m \leq-k-1$ or $m \geq \lambda+k$. For $m=\lambda$, (4.10) gives the normalization

$$
[1] t^{-m} s_{2,(\lambda, 0)}^{k}(s, t)\left(1-\frac{t}{s}\right)^{k-1}\left(1-\frac{s}{t}\right)^{k}=\frac{\lambda !(\lambda+2 k-1) !}{(\lambda+k-1) !(\lambda+k) !} .
$$

Let $\boldsymbol{\mu}=\left(\mu_{1}, \mu_{2}, \ldots, \mu_{n}\right), \mu_{1} \geq \mu_{2} \geq \cdots \geq \mu_{n} \geq 0$, be a partition. Goulden [8] has shown that the Schur functions satisfy the orthogonality

$$
[1] s_{n, \boldsymbol{\lambda}}^{1}\left(t_{1}, \ldots, t_{n}\right) s_{n, \boldsymbol{\mu}}^{1}\left(\frac{1}{t_{1}}, \ldots, \frac{1}{t_{n}}\right) \prod_{1 \leq i<j \leq n}\left(1-\frac{t_{i}}{t_{j}}\right)=\chi(\boldsymbol{\lambda}=\boldsymbol{\mu}) .
$$

This suggests

CONJECTURE 2.

$$
\begin{aligned}
& {[1] s_{n, \lambda}^{k}\left(t_{1}, \ldots, t_{n}\right) s_{n, \mu}^{k}\left(\frac{1}{t_{1}}, \ldots, \frac{1}{t_{n}}\right)} \\
& \quad \cdot \prod_{1 \leq i<j \leq n}\left(1-\frac{t_{j}}{t_{i}}\right)^{k-1}\left(1-\frac{t_{i}}{t_{j}}\right)^{k}=\chi(\lambda=\mu) g_{n, \lambda}^{k},
\end{aligned}
$$

where

$$
g_{n, \lambda}^{k}=(-1)^{k\left(\begin{array}{c}
n \\
2
\end{array}\right)} \prod_{1 \leq i<j \leq n} \frac{\left(\lambda_{i}-\lambda_{j}+k(j-i)\right)_{k}}{\left(\lambda_{j}-\lambda_{i}-k(j-i)\right)_{k}} .
$$

This is known for $n=2$ by (4.11) and (4.12) and for $k=1$ by Goulden's result (4.13). Thus (4.14) combines orthogonality relations for the Schur functions and Jacobi polynomials.

5. A Schur function identity. It is well-known (see Stanley [16]) that the Schur functions (1.5) satisfy (1.12). We extend this by the following theorem.

THEOREM 3. Let $k \geq 1, M \geq 0$. Then

$$
\begin{aligned}
\sum_{\lambda_{1} \geq \lambda_{2} \geq 0} & \frac{\left(2 k+\lambda_{1}-\lambda_{2}\right)_{M}\left(1+\lambda_{1}-\lambda_{2}\right)_{k-1}}{(k)_{M}(k-1) !} s_{2,\left(\lambda_{1}, \lambda_{2}\right)}^{k}(s, t) \\
& =\frac{1}{(1-s t)(1-s)^{k+M}(1-t)^{k+M}} s_{2(M, 0)}^{k}(1-s, 1-t) .
\end{aligned}
$$

ProOF. By (1.4), we have

$$
s_{2,\left(\lambda_{1}, \lambda_{2}\right)}^{k}(s, t)=(s t)^{\lambda_{2}} s_{2,\left(\lambda_{1}-\lambda_{2}, 0\right)}^{k}(s, t) .
$$

Since the coefficient in (5.1) depends on $\lambda_{1}-\lambda_{2}$, we can carry out the sum with respect to $\lambda_{2}$ using (5.2). Setting $\delta=\lambda_{1}-\lambda_{2}$, the left side of (5.1) becomes

$$
\frac{1}{(1-s t)} \sum_{\delta \geq 0} \frac{(2 k+\delta)_{M}(1+\delta)_{k-1}}{(k)_{M}(k-1) !} s_{2,(\delta, 0)}^{k}(s, t) .
$$

Expand $s_{2,(\delta, 0)}^{k}(s, t)$ by $(1.7)$ and set $j=\delta-i$. This equals

$$
\frac{1}{(1-s t)} \sum_{i, j \geq 0} \frac{(2 k+i+j)_{M}(1+i+j)_{k-1}}{(k)_{M}(k-1) !} \frac{(-i-j)_{i}(k)_{i}}{i !(1-i-j-k)_{i}} s^{i} t^{j}
$$


Reversing $(-i-j)_{i}$ and $(1-i-j-k)_{i}$ by (3.12), we have

$$
\frac{(1+i+j)_{k-1}(-i-j)_{i}(k)_{i}}{(k-1) ! i !(1-i-j-k)_{i}}=\frac{(1+i)_{k-1}(1+j)_{k-1}}{(k-1) !(k-1) !} .
$$

Using the Chu-Vandermonde sum (4.2) and (3.12), we obtain

$$
\begin{aligned}
& \sum_{l=0}^{M}(-1)^{l} \frac{(-M)_{l}}{l !}(A)_{l}(B)_{M-l}=(B)_{M} \sum_{l=0}^{M} \frac{(-M)_{l}(A)_{l}}{l !(1-B-M)_{l}} \\
& =(B)_{M} \frac{(1-A-B-M)_{M}}{(1-B-M)_{M}}=(A+B)_{M} .
\end{aligned}
$$

This is an equivalent formulation of (4.2). For $A=k+i, B=k+j$, (5.6) gives

$$
(2 k+i+j)_{M}=\sum_{l=0}^{M}(-1)^{l} \frac{(-M)_{l}}{l !}(k+i)_{l}(k+j)_{M-l} .
$$

Observe that

$$
\begin{gathered}
\frac{(1+i)_{k-1}}{(k-1) !}(k+i)_{l}=\frac{(k-1+l+i) !}{i !(k-1) !}=(k)_{l} \frac{(k+l)_{i}}{i !}, \\
\frac{\left(1_{j}\right)_{k-1}}{(k-1) !}(k+j)_{M-l}=(k)_{M-l} \frac{(k+M-l)_{j}}{j !} \\
(k)_{M-l}=\frac{(k)_{M}}{(k+M-l)_{l}}=(-1)^{l} \frac{(k)_{M}}{(1-M-k)_{l}} .
\end{gathered}
$$

Substitute (5.7) into (5.4) and simplify using (5.5) and (5.8). (5.4) becomes

$$
\frac{1}{(1-s t)} \sum_{l=0}^{M} \frac{(-M)_{l}(k)_{l}}{l !(1-M-k)_{l}} \sum_{i, j \geq 0} \frac{(k+l)_{i}}{i !} s^{i} \frac{(k+M-l)_{j}}{j !} t^{j} .
$$

Putting $c=-n / x$ in the Chu-Vandermonde sum (4.2) and letting $n$ tend to $\infty$ gives the well-known binomial theorem

$$
\sum_{i \geq 0} \frac{(b)_{i}}{i !} x^{i}=\frac{1}{(1-x)^{b}}
$$

valid if the left side terminates or $|x|<1$. We may carry out the summations in (5.9) with respect to $i$ and $j$. We find that (5.9) equals

$$
\begin{aligned}
\frac{1}{(1-s t)} \sum_{l \geq 0} & \frac{(-M)_{l}(k)_{l}}{l !(1-M-k)_{l}} \frac{1}{(1-s)^{k+l}(1-t)^{k+M-l}} \\
& =\frac{1}{(1-s t)(1-s)^{k+M}(1-t)^{k+M}} s_{2,(M, 0)}^{k}(1-s, 1-t)
\end{aligned}
$$

as required.

6. A $q$-difference equation. Fix $q$ with $0<q<1$ and set

$$
\begin{aligned}
(a)_{0} & =(a ; q)_{0}=1, \\
(a)_{m} & =(a ; q)_{m}=\prod_{i=0}^{m-1}\left(1-a q^{i}\right), \quad m \geq 1, \\
(a)_{\infty} & =(a ; q)_{\infty}=\lim _{m \rightarrow \infty}(a ; q)_{m}=\prod_{i=0}^{\infty}\left(1-a q^{i}\right) .
\end{aligned}
$$


Following Jackson [11], we define the $q$-gamma function

$$
\Gamma_{q}(x)=\frac{(q)_{\infty}}{\left(q^{x}\right)_{\infty}}(1-q)^{(1-x)}
$$

and the $q$-integral

$$
\int_{0}^{a} f(t) d_{q} t=a(1-q) \sum_{n=0}^{\infty} q^{n} f\left(a q^{n}\right)
$$

A $q$-analogue of the beta integral (1.9) is given by

$$
\int_{0}^{1} t^{(x-1)} \frac{(q t)_{\infty}}{\left(q^{y} t\right)_{\infty}} d_{q} t=\frac{\Gamma_{q}(x) \Gamma_{q}(y)}{\Gamma_{q}(x+y)} .
$$

This is equivalent to the well-known $q$-binomial theorem. See Askey [3].

Hahn [10] has shown that the polynomials

$$
{ }_{q} p_{n}^{(x, y)}(t)=\sum_{i=0}^{n} \frac{\left(q^{-n}\right)_{i}\left(q^{n+x+y-1)}\right)_{i}}{(q)_{i}\left(q^{x}\right)_{i}}(q t)^{i}
$$

are orthogonal with respect to the $q$-beta distribution (6.4). These are called the little $q$-Jacobi polynomials. They satisfy the orthogonality relation

$$
\int_{0}^{1} t^{m}{ }_{q} p_{n}^{(x, y)}(t) t^{(x-1)} \frac{(q t)_{\infty}}{\left(q^{y} t\right)_{\infty}} d_{q} t=0, \quad 0 \leq m<n .
$$

Using

$$
\Gamma_{q}(x+1)=\frac{\left(1-q^{x}\right)}{(1-q)} \Gamma_{q}(x)
$$

this becomes

$$
\sum_{i=0}^{n}{ }_{q} c_{n}^{(x, y)}(i)\left(q^{x+m}\right)_{i}\left(q^{x+y+m+i}\right)_{n-i}=0, \quad 0 \leq m<n
$$

where

$$
{ }_{q} c_{n}^{(x, y)}(i)=q^{i} \frac{\left(q^{-n}\right)_{i}\left(q^{n+x+y-1}\right)_{i}}{(q)_{i}\left(q^{x}\right)_{i}} .
$$

The coefficients (6.9) are uniquely determined by the orthogonality (6.8) and the normalization

$$
{ }_{q} c_{n}^{(x, y)}(0)=1
$$

Recall [12] that

$$
{ }_{q} \Delta_{n}^{2 k}(\mathbf{t})=\prod_{1 \leq i<j \leq n} t_{i}^{(2 k-1)}\left(q^{1-k} \frac{t_{j}}{t_{i}}\right)_{(2 k-1)}\left(t_{i}-t_{j}\right)
$$

is a symmetric $q$-analogue of $\prod_{1 \leq i<n \leq n}\left(t_{i}-t_{j}\right)^{2 k}$. We have the symmetric measure

$$
{ }_{q} W_{n}^{\prime}(x, y, k)=\prod_{i=1}^{n} t_{i}^{(x-1)} \frac{\left(q t_{i}\right)_{\infty}}{\left(q^{y} t_{i}\right)_{\infty}}{ }_{q} \Delta_{n}^{2 k}(\mathbf{t}) .
$$


In [12], we have a $q$-analogue of (1.1). It is

CONJECTURE 4 . Let $n \geq 2, k \geq 0$. For each partition $\lambda=\left(\lambda_{1}, \lambda_{2}, \ldots, \lambda_{n}\right)$, $\lambda_{1} \geq \lambda_{2} \geq \cdots \geq \lambda_{n} \geq 0$, there is a homogeneous symmetric polynomial ${ }_{q} s_{n, \lambda}^{k}(\mathbf{t})$ with leading term $\prod_{i=1}^{n} t_{i}^{\lambda_{i}}$ such that

$$
\begin{aligned}
\int_{0}^{1} \cdots & \int_{0}^{1}{ }_{q} s_{n, \lambda}^{k}(\mathbf{t}){ }_{q} W_{n}^{\prime}(x, y, k) d_{q} t_{1} \cdots d_{q} t_{n} \\
= & n ! q\left[q^{\left[k\left(\sum_{i=1}^{n}(i-1) \lambda_{i}\right)+k x\left(\begin{array}{c}
n \\
2
\end{array}\right)+2 k^{2}\left(\begin{array}{c}
n \\
3
\end{array}\right)\right]}\right. \\
& \cdot{ }_{q} f_{n, \lambda}^{k} \prod_{i=1}^{n} \frac{\Gamma_{q}\left(x+(n-i) k+\lambda_{i}\right) \Gamma_{q}(y+(n-i) k)}{\Gamma_{q}\left(x+y+(2 n-i-1) k+\lambda_{i}\right)},
\end{aligned}
$$

where

$$
{ }_{q} f_{n, \lambda}^{k}=\prod_{1 \leq i<j \leq n} \frac{\left(q^{\lambda_{i}+\lambda_{j}+k(j-i)}\right)_{k}}{(1-q)^{k}}
$$

We call these the $q$-Solberg polynomials.

Kadell [12] has shown that for $k=1$, the $q$-Selberg polynomials are the Schur functions

$$
{ }_{q} s_{n, \lambda}^{1}(\mathbf{t})=s_{n, \lambda}^{1}(\mathbf{t})
$$

as happens (1.5) when $q=1$.

For $n=2$, we take $\lambda_{1}=\lambda, \lambda_{2}=0, s=t_{1}, t=t_{2}$. (6.13) and (6.14) become

$$
\begin{aligned}
\int_{0}^{1} \int_{0}^{1}{ }_{q} s_{2,(\lambda, 0)}^{k}(s, t)_{q} W_{2}^{\prime}(x, y, k) d_{q} s d_{q} t \\
\quad=2 q^{k x} \frac{\left(q^{\lambda+k}\right)_{k}}{(1-q)^{k}} \frac{\Gamma_{q}(x) \Gamma_{q}(x+k+\lambda) \Gamma_{q}(y) \Gamma_{q}(y+k)}{\Gamma_{q}(x+y+k) \Gamma_{q}(x+y+2 k+\lambda)}
\end{aligned}
$$

Observe that

$$
\left(s-q^{-k} t\right)\left(s-q^{k} t\right){ }_{q} W_{2}^{\prime}(x, y, k)={ }_{q} W_{2}^{\prime}(x, y, k+1)
$$

and

$$
\begin{aligned}
& \left(1-q^{x+k+\lambda+1}\right)\left(1-q^{x+y+k}\right)-q^{x}\left(1-q^{\lambda+k+1}\right)\left(1-q^{y+k}\right) \\
& =\left(1-q^{x}\right)\left(1-q^{x+y+2 k+\lambda+1}\right) .
\end{aligned}
$$


Using (6.16), (6.17), and (6.18), we obtain

(6.19)

$$
\begin{aligned}
\int_{0}^{1} \int_{0}^{1}\left[{ }_{q} s_{2,(\lambda+2,0)}^{k}(s, t)-\left(s-q^{-k} t\right)\left(s-q^{k} t\right)_{q} s_{2,(\lambda, 0)}^{k+1}(s, t)\right]_{q} W_{2}^{\prime}(x, y, k) d_{q} s d_{q} t \\
=2 q^{k z} \frac{\left(q^{\lambda+k+2}\right)_{k}}{(1-q)^{k}} \frac{\Gamma_{q}(x) \Gamma_{q}(x+k+\lambda+2) \Gamma_{q}(y) \Gamma_{q}(y+k)}{\Gamma_{q}(x+y+k) \Gamma_{q}(x+y+2 k+\lambda+2)} \\
\quad-2 q^{(k+1) x} \frac{\left(q^{\lambda+k+1}\right)_{(k+1)}}{(1-q)^{k+1}} \frac{\Gamma_{q}(x) \Gamma_{q}(x+k+\lambda+1) \Gamma_{q}(y) \Gamma_{q}(y+k+1)}{\Gamma_{q}(x+y+k+1) \Gamma_{q}(x+y+2 k+\lambda+2)} \\
=2 q^{k x} \frac{\left(q^{\lambda+k+2}\right)_{k}}{(1-q)^{k}} \frac{\Gamma_{q}(x) \Gamma_{q}(x+k+\lambda+1) \Gamma_{q}(y) \Gamma_{q}(y+k)}{\Gamma_{q}(x+y+k+1) \Gamma_{q}(x+y+2 k+\lambda+2)} \\
\quad \cdot\left(\frac{\left(1-q^{x+k+\lambda+1}\right)\left(1-q^{x+y+k}\right)}{(1-q)(1-q)}-q^{x} \frac{\left(1-q^{\lambda+k+1}\right)(y+k)}{(1-q)(1-q)}\right) \\
=2 q^{k x} \frac{\left(q^{\lambda+k+2}\right)_{k}}{(1-q)^{k}} \frac{\Gamma_{q}(x+1) \Gamma_{q}(x+k+\lambda+1) \Gamma_{q}(y) \Gamma_{q}(y+k)}{\Gamma_{q}(x+y+k+1) \Gamma_{q}(x+y+2 k+\lambda+1)} .
\end{aligned}
$$

(5.2) and (6.16) give

$$
\begin{aligned}
& \int_{0}^{1} \int_{0}^{1} s t_{q} s_{2,(\lambda, 0)}^{k}(s, t)_{q} W_{2}^{\prime}(x, y, k) d_{q} s d_{q} t \\
& \quad=2 q^{k(x+1)} \frac{\left(q^{\lambda+k}\right)_{k}}{(1-q)^{k}} \frac{\Gamma_{q}(x+1) \Gamma_{q}(x+k+\lambda+1) \Gamma_{q}(y) \Gamma_{q}(y+k)}{\Gamma_{q}(x+y+k+1) \Gamma_{q}(x+y+2 k+\lambda+1)} .
\end{aligned}
$$

Comparing (6.19) and (6.20), we have

$$
\begin{gathered}
\int_{0}^{1} \int_{0}^{1}\left[{ }_{q} s_{2,(\lambda+2,0)}^{k}(s, t)-\left(s-q^{-k} t\right)\left(s-q^{k} t\right)_{q} s_{2,(\lambda, 0)}^{k+1}(s, t)\right]{ }_{q} W_{2}^{\prime}(x, y, k) d_{q} s d_{q} t \\
\quad=q^{-k} \frac{\left(q^{\lambda+2 k}\right)_{2}}{\left(q^{\lambda+k}\right)_{2}} \int_{0}^{1} \int_{0}^{1} s t_{q} s_{2,(\lambda, 0)}^{k}(s, t)_{q} W_{2}^{\prime}(x, y, k) d_{q} s d_{q} t .
\end{gathered}
$$

Equating integrands and rearranging gives

$$
\begin{aligned}
& \left(q^{\lambda+2 k}\right)_{2} s t_{q} s_{2,(\lambda, 0)}^{k}(s, t) \\
& \quad=q^{k}\left(q^{\lambda+k}\right)_{2}\left[{ }_{q} s_{2,(\lambda+2,0)}^{k}(s, t)-\left(s-q^{-k} t\right)\left(s-q^{k} t\right)_{q} s_{2,(\lambda, 0)}^{k+1}(s, t)\right] .
\end{aligned}
$$

A few computations suggest

THEOREM 5. For $k \geq 1$,

$$
{ }_{q} s_{2,(\lambda, 0)}^{k}(s, t)=\sum_{i=0}^{\lambda} \frac{\left(q^{-\lambda}\right)_{i}\left(q^{k}\right)_{i}}{(q)_{i}\left(q^{1-\lambda-k}\right)_{i}}\left(q^{1-k} s\right)^{i} t^{\lambda-i} .
$$

By (6.5) this is

$$
{ }_{q} s_{2,(\lambda, 0)}^{k}(s, t)=t^{\lambda}{ }_{q} p_{\lambda}^{(1-\lambda-k, 2 k)}\left(q^{-k} s / t\right), \quad k \geq 1 .
$$

Thus for $n=2$, the $q$-Selberg polynomials are little $q$-Jacobi polynomials. Divide both sides of (6.22) by $\left(q^{\lambda+2 k}\right)_{2} s t^{\lambda+1}$ and replace $q^{-k} s / t$ by $t$. Using (6.24), (6.22) 
becomes

$$
\begin{aligned}
{ }_{q} p_{\lambda}^{(1-\lambda-k, 2 k)}(t)= & \frac{\left(q^{\lambda+k}\right)_{2}}{\left(q^{\lambda+2 k}\right)_{2}} \frac{1}{t}{ }_{q} p_{\lambda+2}^{(-1-\lambda-k, 2 k)}(t) \\
& -\frac{\left(q^{\lambda+k}\right)_{2}}{\left(q^{\lambda+2 k}\right)_{2}} \frac{1}{t}(1-t)\left(1-q^{2 k} t\right)_{q} p_{\lambda}^{(-\lambda-k, 2 k+2)}\left(\frac{t}{q}\right) .
\end{aligned}
$$

7. A proof of Theorem 5. We mimic the proof of the case $q=1$. We may show that (6.23) satisfies (6.22) and proceed by induction on $k$. We use (6.25) rather than (6.22). By (3.1) and (6.15), we see that (6.23) holds for $k=1$.

We now show that for $k \geq 1$, the right side of $(6.25)$ is a polynomial of degree at most $\lambda$ which satisfies the orthogonality (6.8) with the parameters given in (3.2) and the normalization (6.10). Let $a$ be an integer with $\operatorname{Re}(x+a)>0$ and $b, c$, be nonnegative integers. Then

$$
\begin{aligned}
\int_{0}^{1} t^{m}\left(t^{a}\left(q^{1-b} t\right)_{b}\left(q^{y} t\right)_{c q} p_{n}^{(x+a, y+b+c)}\left(q^{-b} t\right)\right) t^{(x-1)} \frac{(q t)_{\infty}}{\left(q^{y} t\right)_{\infty}} d_{q} t & =0 \\
& 0 \leq m<n .
\end{aligned}
$$

To see this observe that

$$
\left(q^{1-b} t\right)_{b} \frac{(q t)_{\infty}}{\left(q^{y} t\right)_{\infty}}\left(q^{y} t\right)_{c}=\frac{\left(q^{1-b} t\right)_{\infty}}{\left(q^{y+c} t\right)_{\infty}}
$$

and that this vanishes when $t=q^{v}, 0 \leq v<b$. The substitution $t=q^{b} s, d_{q} t=$ $q^{b} d_{q} s$, gives

$$
\int_{0}^{q^{b}} f\left(q^{-b} t\right) d_{q} t=q^{b} \int_{0}^{1} f(s) d_{q} s
$$

in agreement with (6.3). The left side of (7.1) equals

$$
\begin{aligned}
& \int_{0}^{q^{b}} t^{m}{ }_{q} p_{n}^{(x+a, y+b+c)}\left(q^{-b} t\right) t^{(x+a-1)} \frac{\left(q^{1-b} t\right)_{\infty}}{\left(q^{y+c} t\right)_{\infty}} d_{q} t \\
& =q^{b(x+m+a)} \int_{0}^{1} s^{m}{ }_{q} p_{n}^{(x+a, y+b+c)}(s) s^{(x+a+1)} \frac{(q s)_{\infty}}{\left(q^{y+b+c} s\right)_{\infty}} d_{q} s
\end{aligned}
$$

which is 0 for $0 \leq m<n$. Set

$$
t^{a}\left(q^{1-b} t\right)_{b}\left(q^{y} t\right)_{c q} p_{n}^{(x+a, y+b+c)}\left(q^{-b} t\right)=\sum_{i=a}^{a+b+c+n}{ }_{q} d_{n,(a, b, c)}^{(x, y)}(i) t^{i}
$$

The orthogonality (7.1) becomes

$$
\begin{aligned}
\sum_{i=a}^{a+b+c+n}{ }_{q} d_{n,(a, b, c)}^{(x, y)}(i)\left(q^{x+m+a}\right)_{(i-a)}\left(q^{x+y+m+i}\right)_{(n+a+b+c-i)}=0, & \\
& 0 \leq m<n .
\end{aligned}
$$

Substituting (6.5) into (7.5), we see that both sides of (7.6) are rational functions of $q^{x}$ and $q^{y}$. Setting

$$
x=1-\lambda-k, \quad y=2 k, \quad n=\lambda, \quad a=-1, \quad b=c=1,
$$


in (7.6), we obtain

$$
\sum_{i=-1}^{\lambda+1} q d_{\lambda,(-1,1,1)}^{(1-\lambda-k, 2 k)}(i)\left(q^{-\lambda-k+m}\right)_{(i+1)}\left(q^{1-\lambda+k+m+i}\right)_{(\lambda+1-i)}=0
$$$$
0 \leq m<\lambda
$$

Using

$$
x=-\lambda-k, \quad y=2 k, \quad n=\lambda+2, \quad a=-1, \quad b=c=0,
$$

we obtain

$$
\begin{aligned}
& \sum_{i=-1}^{\lambda+1} q d_{\lambda+2,(-1,0,0)}^{(-\lambda-k, 2 k)}(i)\left(q^{-1-\lambda-k+m}\right)_{(i+1)}\left(q^{-\lambda+k+m+i}\right)_{(\lambda+1-i)}=0 \\
& 0 \leq m<\lambda+2 .
\end{aligned}
$$

If we replace $m$ by $m+1$ in (7.10), we obtain an orthogonality with the same coefficients as in (7.8) which is valid for $-1 \leq m<\lambda+1$. Let

$$
\text { right side of }(6.25)=\sum_{i=-1}^{\lambda+1} q e(i) t^{i} \text {. }
$$

Then we have

$$
\sum_{i=-1}^{\lambda+1} q e(i)\left(q^{-\lambda-k+m}\right)_{(i+1)}\left(q^{1-\lambda+k+m+i}\right)_{(\lambda+1-i)}=0, \quad 0 \leq m<\lambda .
$$

Using

$$
(\alpha)_{i}=(-\alpha)^{i} q^{\left(\begin{array}{c}
i \\
2
\end{array}\right)}\left(\frac{q^{1-i}}{\alpha}\right)
$$

we have, for $k \geq 1$,

$$
q^{(\lambda-i)(1-k)} \frac{\left(q_{\lambda-i}^{-\lambda}\left(q^{k}\right)_{\lambda-i}\right.}{(q)_{\lambda-i}\left(q^{1-\lambda-k}\right)_{\lambda-i}}=q^{i(1-k)} \frac{\left(q^{-\lambda}\right)_{i}\left(q^{k}\right)_{i}}{(q)_{i}\left(q^{1-\lambda-k}\right)_{i}}
$$

Thus ${ }_{q} s_{2,(\lambda, 0)}^{k}(s, t)$ and hence the right side of (6.22) are both symmetric in $s$ and $t$. This gives

$$
\begin{gathered}
{ }_{q} e(i)=q^{(2 i-\lambda) k}{ }_{q} e(\lambda-i), \quad-1 \leq i \leq \lambda+1, \\
{ }_{q} e(-1)={ }_{q} e(\lambda+1)=0 .
\end{gathered}
$$

By (7.15), the sum in (7.12) may be taken with $i$ running from 0 to $\lambda$. Dividing by $\left(1-q^{-\lambda-k+m}\right)\left(1-q^{1+k+m}\right)$, we obtain

$$
\sum_{i=0}^{\lambda} q_{q} e(i)\left(q^{1-\lambda-k+m}\right)_{i}\left(q^{1-\lambda+k+m+i}\right)_{(\lambda-i)}=0, \quad 0 \leq m<\lambda .
$$

Hence the right side of $(6.25)$ is a polynomial of degree at most $\lambda$ which satisfies the orthogonality relation (6.8) with the parameters given by (3.2). By (6.8), the left side of (6.25) also satisfies (7.16). A simple computation

$$
\frac{\left(q^{\lambda+k}\right)_{2}}{\left(q^{\lambda+2 k}\right)_{2}}\left[q \frac{\left(1-q^{-\lambda-2}\right)\left(1-q^{k}\right)}{(1-q)\left(1-q^{-1-\lambda-k}\right)}-\frac{\left(1-q^{-\lambda}\right)\left(1-q^{k+1}\right)}{(1-q)\left(1-q^{-\lambda-k}\right)}+1+q^{2 k}\right]=1
$$

gives the normalization $(6.10)$. 
To complete the proof of Theorem 5 , we must show that the system of equations (7.16) and the normalization (7.17) uniquely determine ${ }_{q} p_{\lambda}^{(1-\lambda-k, 2 k)}(t)$. Substituting (6.10) into (6.8) gives a system of equations with coefficient matrix

$$
{ }_{q} C_{n}^{(x, y)}=\left|\left(q^{x+i}\right)_{j}\left(q^{x+y+i+j}\right)_{n-j}\right|_{0 \leq i<n, 1 \leq j \leq n} .
$$

For $1 \leq s \leq n$, set

$$
{ }_{q} C_{n, s}^{(x, y)}=\left|{ }_{q} c_{n, s}^{(x, y)}(i, j)\right|_{0 \leq i<n, 1 \leq j \leq n}
$$

where

$$
{ }_{q} c_{n, s}^{(x, y)}(i, j)= \begin{cases}\left(q^{x+i}\right)_{j}\left(q^{x+y+i+j+n-s}\right)_{s-j}, & 0 \leq i<n, 1 \leq j \leq s \\ \left(q^{x+i}\right)_{j}, & 0 \leq i<n, s<j \leq n\end{cases}
$$

Then we have

$$
{ }_{q} C_{n}^{(x, y)}={ }_{q} C_{n, n}^{(x, y)}
$$

Observe that

$$
\begin{aligned}
& \left(q^{x+i}\right)_{j}\left(q^{x+y+i+j+n-s}\right)_{s-j}-q^{y+n-s}\left(q^{x+i}\right)_{j+1}\left(q^{x+y+i+j+n-s+1}\right)_{s-j-1} \\
& \quad=\left(1-q^{y+n-s}\right)\left(q^{x+i}\right)_{j}\left(q^{x+y+i+j+n-s+1}\right)_{s-j-1}
\end{aligned}
$$

Using (7.22), we may subtract $q^{y+n-s}$ times column $j+1$ from column $j$ and divide by $\left(1-q^{y+n-s}\right)$ where $j$ runs from 1 to $s-1$. This gives

$$
\operatorname{det}\left({ }_{q} C_{n, s}^{(x, y)}\right)=\left(1-q^{y+n-s}\right)^{(s-1)} \operatorname{det}\left({ }_{q} C_{n, s-1}^{(x, y)}\right), \quad 2 \leq s \leq n .
$$

To compute the determinant of

$$
{ }_{q} C_{n, 1}^{(x, y)}=\left|\left(q^{z+i}\right){ }_{j}\right|_{0 \leq i<n, 1 \leq j \leq n},
$$

divide row $i$ by $\left(1-q^{x+i}\right)$ for $0 \leq i<n$. This gives

$$
\operatorname{det}\left({ }_{q} C_{n, 1}^{(x, y)}\right)=\left(q^{x}\right)_{n} \operatorname{det}\left({ }_{q} D_{n}^{(x, y)}\right),
$$

where

$$
{ }_{q} D_{n}^{(x, y)}=\left|\left(q^{x+i+1}\right)_{j-1}\right|_{0 \leq i<n, 1 \leq j \leq n} .
$$

We may use

$$
\left(q^{x+i+1}\right)_{j-1}-\left(q^{x+i}\right)_{j-1}=q^{x+i}\left(1-q^{j-1}\right)\left(q^{x+i+1}\right)_{j-2}
$$

to subtract row $i-1$ from row $i$ where $i$ runs from $n-1$ to 1 and then expand along column 1 . We obtain

$$
\begin{aligned}
\operatorname{det}\left({ }_{q} D_{n}^{(x, y)}\right) & =\operatorname{det}\left|\begin{array}{ccccc}
1 & \left(1-q^{x+1}\right) & \left(q^{x+1}\right)_{2} & \ldots & \left(q^{x+1}\right)_{n-1} \\
1 & \left(1-q^{x+2}\right) & \left(q^{x+2}\right)_{2} & \ldots & \left(q^{x+2}\right)_{n-1} \\
\vdots & \vdots & \vdots & & \vdots \\
1 & \left(1-q^{x+n}\right) & \left(q^{x+n}\right)_{2} & \ldots & \left(q^{x+n}\right)_{n-1}
\end{array}\right|_{n \times n} \\
& =\operatorname{det}\left|\begin{array}{cccc}
1 & \left(1-q^{x+1}\right) & \ldots & \\
0 & q^{x+1}(1-q) & \ldots & q^{x+1}\left(1-q^{n-1}\right)\left(q^{x+2}\right)_{n-2} \\
\vdots & \vdots & & \vdots \\
0 & q^{x+n-1}(1-q) & \ldots & q^{x+n-1}\left(1-q^{n-1}\right)\left(q^{x+n}\right)_{n-2}
\end{array}\right|_{n \times n} \\
& =q^{\left[x(n-1)+\left(\begin{array}{c}
n \\
2
\end{array}\right)\right]}(q)_{n-1} \operatorname{det}\left({ }_{q} D_{n-1}^{(x+1, y)}\right)
\end{aligned}
$$


Observe that

$$
{ }_{q} P_{n}(x)=q^{\left[x\left(\begin{array}{c}
n \\
2
\end{array}\right)+\left(\begin{array}{c}
n+1 \\
3
\end{array}\right)+\left(\begin{array}{c}
n \\
3
\end{array}\right)\right]}
$$

satisfies

$$
{ }_{q} P_{n}(x)=q^{\left[x(n-1)+\left(\begin{array}{c}
n \\
2
\end{array}\right)\right]}{ }_{q} P_{n-1}(x+1) .
$$

Combining our results, we obtain

$$
\operatorname{det}\left({ }_{q} C_{n}^{(x, y)}\right)=q^{\left[x\left(\begin{array}{c}
n \\
2
\end{array}\right)+\left(\begin{array}{c}
n+1 \\
3
\end{array}\right)+\left(\begin{array}{c}
n \\
3
\end{array}\right)\right]}\left(q^{x}\right)_{n} \prod_{s=2}^{n}(q)_{s-1}\left(1-q^{y+n-s}\right)^{(s-1)} .
$$

Substituting (3.2) into (7.31) gives

$$
\begin{aligned}
\operatorname{det}\left({ }_{q} C_{\lambda}^{(1-\lambda-k, 2 k)}\right)= & q^{\left[(1-\lambda-k)\left(\begin{array}{c}
\lambda \\
2
\end{array}\right)+\left(\begin{array}{c}
\lambda+1 \\
3
\end{array}\right)+\left(\begin{array}{c}
\lambda \\
3
\end{array}\right)\right]} \\
& \cdot\left(q^{1-\lambda-k}\right)_{\lambda} \prod_{s=2}^{\lambda}(q)_{(s-1)}\left(1-q^{2 k+\lambda-s}\right)^{(s-1)} .
\end{aligned}
$$

The proof of Theorem 5 is now completed by observing that for $k \geq 1$ this is not 0 .

8. Epilogue. There are many measures $d F(t)$ which provide $q$-analogues of the beta integral (1.9) and for which there is a conjectured $q$-analogue of Selberg's integral. See Askey [4] and Rahman [14]. Kadell [13] and Habsieger [9] have independently proven Conjecture 1 of Askey [4], which is based upon (6.4).

In [12], we were able to treat the case $k=1$ by a method which seems to work for many of these measures. We may obtain the same results by using the integral representation

$$
P_{n}(t)=\int \cdots \int \prod_{i=1}^{n}\left(t_{i}-t\right) \prod_{1 \leq i<j \leq n}\left(t_{i}-t_{j}\right)^{2} d F\left(t_{1}\right) \cdots d F\left(t_{n}\right)
$$

of the polynomials $\left\{P_{n}(t) \mid n \geq 0\right\}$ which are orthogonal with respect to $d F(t)$. See Szegö [17, (2.2.10)]. Aomoto [2] extends (8.1) for the beta distribution (1.9). A $q$-analogue of this result for this measure (6.4) follows from Kadell's $q$-analogue [13] of (1.6).

Compare the analysis of $\S \S 6$ and 7 with that of $\S \S 2$ and 3 . This suggests that Theorem 1 (1.7) can be extended using the orthogonality of $\left\{P_{n}(t) \mid n \geq 0\right\}$. Rahman [14] uses a different method to treat the case $n=2$ of his conjecture. See Askey and Ismail [5], Askey and Wilson [6], and Andrews and Askey [1] for many orthogonal polynomials.

\section{REFERENCES}

1. G. E. Andrews and R. A. Askey, q-analogueues of the classical orthogonal polynomials and applications (to appear).

2. K. Aomoto, Jacobi polynomials associated with Selberg integrals, SIAM J. Math. Anal. 18 (1987), 545-549.

3. R. Askey, The q-gamma and q-beta functions, Appl. Anal. 8 (1978), 125-141.

4. S_ Some basic hypergeometric extensions of integrals of Selberg and Andrews, SIAM J. Math. Anal. 11 (1980), 938-951.

5. R. Askey and M. Ismail, Recurrence relations, continued fractions and orthogonal polynomials, Mem. Amer. Math. Soc., \# 300, 1984. 
6. R. Askey and J. Wilson, Some basic hypergeometric orthogonal polynomials that generalize Jacobi polynomials, Mem. Amer. Math. Soc., \# 319, 1985.

7. W. N. Bailey, Generalized hypergeometric series, Cambridge Math. Tract \# 32, Cambridge Univ. Press, Cambridge, 1935 (reprinted by Hafner, New York, 1964).

8. I. P. Goulden, private communication.

9. L. Habsieger, Une q-intégrale de Selberg-Askey, SIAM J. Math. Anal. (to appear).

10. W. Hahn, Über polynome, die gleichzeitig zwei verscheidenen Orthogonalsystemen angehören, Math. Nachr. 2 (1949), 263-278.

11. F. H. Jackson, On q-definite integrals, Quart. J. Pure Appl. Math. 41 (1910), 193-203.

12. K. W. J. Kadell, $A$ proof of some $q$-analogues of Selberg's integral for $k=1$, SIAM J. Math. Anal. 19 (1988), 944-968.

13. __ A proof of Askey's conjectured q-analogue of Selberg's integral and a conjecture of Morris, SIAM J. Math. Anal. 19 (1988), 969-986.

14. M. Rahman, Another conjectured q-Selberg integral, SIAM J. Math. Anal. 17 (1986), 12671279.

15. A. Selberg, Bemerkninger om et multipelt integral, Norsk. Mat. Tiddskr. 26 (1944), 71-78.

16. R. P. Stanley, Theory and application of plane partitions, Parts 1 and 2, Stud. Appl. Math. 50 (1971), 167-188 and 259-279.

17. G. Szegö, Orthogonal polynomials, 4th ed., Amer. Math. Soc. Colloq. Publ., vol. 23, Amer. Math. Soc., Providence, R.I., 1975.

Department of Mathematics, Arizona State University, Tempe, Arizona 85287 